\title{
PRP :A MIRACLE FOR CHRONIC ULCERS
}

\section{Orthopaedics}

Dr. Sahil Kanwar Senior Resident, Department of orthopaedics, SRMSIMS.

\section{Dr. Neni Agarwal* Senior Resident, Department of dermatology, SRMSIMS. *Corresponding Author}

\section{ABSTRACT}

In the recent years, the use of platelet rich plasma (PRP) has increased notably in a range of diseases and settings, such as wound healing. In this study we intend to likewise, a total of 25 patients with chronic non healing ulcers preferably on the lower leg and feet were enrolled and treated with PRP prepared from their own blood at a weekly interval for 1 month along with daily dressing. Response assessed after 1 month showed significant improvement in the ulcer symptoms and dimensions.

\section{KEYWORDS}

\section{PRP, chronic ulcer}

\section{INTRODUCTION}

Platelet-rich plasma (PRP) (syn. Autologous platelet gel, plasma-rich growth factors and platelet-concentrated plasma) means "abundant platelets that are concentrated into a small volume of plasma." The pivotal discovery of platelet-derived growth factor (PDGF) in promoting wound healing, angiogenesis and tissue remodelling threw light on this novel autologous therapeutic modality.

\section{Chronic Ulcer}

Consensus has been reached such that a wound may be defined as chronic if complete healing has not been achieved in 6 weeks or no positive response to a treatment change is observed.

Chronic cutaneous wound etiology is diverse. Leg ulcers, mainly secondary to venous insufficiency, are the most frequent. Other highly prevalent lesions include arterial, pressure, neuropathic ulcers, essentially secondary to diabetes or lepra reaction, and Martorell hypertensive ischemic leg ulcer.

The biochemical microenvironment that promotes the chronicity of these lesions is characterized by an abnormally prolonged inflamm atory phase, due to an increase in proinflammatory cytokines and high metalloprotease activity.

Hypoxia and repeated infections promote this excessive amount of proteolytic enzymes and consequent growth factors and fibrin deficit, which impairs wound healing.

An effective intervention must thus modify this environment that impedes healing, and is essential to induce the reparative phase of healing and shorten the prior inflammatory phase.

In order to succeed, chronic-ulcer managementshould have a dual approach, including both underlying disease and local wound treatment.

During the physiological wound-healing process, platelets are activated in the injury site, and growth factors and cytokines are released over time during fibrin retraction. These proteins can bind to the fibrin matrix and to proteoglycans in the extracellular matrix, with the consequent establishment of a storage pool that can be secondarily released by proteinases. ${ }^{3}$

The major families of growth factors that are released from platelets and are involved in wound healing are as follows: ${ }^{4}$

- EGF - stimulates fibroblasts to secrete collagenases, in order to degrade the extracellular matrix during the remodeling phase; encourages keratinocyte and fibroblast proliferation

- TGF

- TGF $\alpha$ - mitogenic and chemotactic for keratinocytes and fibroblasts

- TGF $\beta 1$ and TGF $\beta 2$ - promote angiogenesis, upregulate collagen production, inhibit degradation, stimulate chemoattraction of inflammatory cells.

- VEGF - stimulates angiogenesis during tissue hypoxia
- FGF - promotes angiogenesis, granulation, and epithelialization via endothelial cell, fibroblast, and keratinocyte migration, respectively

- Platelet-derived growth factor (PDGF) - enhances migration of macrophages and fibroblasts; promotes collagen and proteoglycan synthesis.

Considering that PRP is a source of growth factors, and consequently has mitogenic, angiogenic, and chemotactic properties, it represents an interesting alternative adjunctive treatment for recalcitrant wounds.

\section{What Is PRP?}

After learning about what chronic ulcers are and what treatment modality should be opted to treat the ulcer we must learn about the modality which forms the basis of this study- platelet rich plasma (PRP).

PRP is an effective concentration of multiple fundamental growth factors (GFs) by virtue of platelets alone (stored as alpha -granules in platelets) and plasma proteins, namely fibrin, fibronectin and vitronectin.

This cocktail of GFs is pivotal in modulation of tissue repair and regeneration, ${ }^{5}$ whereas the plasma proteins act as a scaffold for the bone, connective tissue and epithelial migration.

Degranulation of the pre-packaged GFs in plateletsoccurs upon "activation" i.e., on coming in contact with coagulation triggers. The secreted GFs in turn bind to their respective transmembrane receptors expressed over adult mesenchymal stem cells, osteoblasts, fibroblasts, endothelial cells, and epidermal cells. ${ }^{6}$ This further induces an internal signal-transduction pathway, unlocking the expression of a normal gene sequence of a cell like cellular proliferation, matrix formation, osteoid production, collagen synthesis, etc., thereby augmenting the natural wound-healing process.

\section{How Is PRP Prepared?}

In a day care setting PRP can be prepared manually or automated devices by a single or a double spin method.

The patient's own (autologous ) blood is collected in ACD-A (acid citrate dextrose) vial and then subjected to centrifugation at a preferable temperature of 20-22degree celcius.

The American Association of Blood Banks technical manual, ${ }^{8}$ states that "platelet-rich plasma is separated from whole blood by 'light-spin' centrifugation and subsequently the platelets are concentrated by 'heavy-spin' centrifugation with removal of the supernatant plasma."

The basic principle behind the PRP separation procedure is as follows.

The centrifugation process separates blood components owing to their different specific gravities.

The first centrifugation is slow to avoid spinning down platelets and to isolate plasma. Platelets are mostly concentrated right on top of the buffy coat layer. Subsequent centrifugation is faster, so that platelets 
are spun down and separate as a pellet at the bottom of the tube from platelet-poor plasma (PPP) above. The final platelet concentration depends on the volume reduction of PPP. Approximately $3 / 4$ of the supernatant is discarded and the platelet-rich pellet is resuspended in remaining amount of plasma. The resulting suspension is used as PRP.

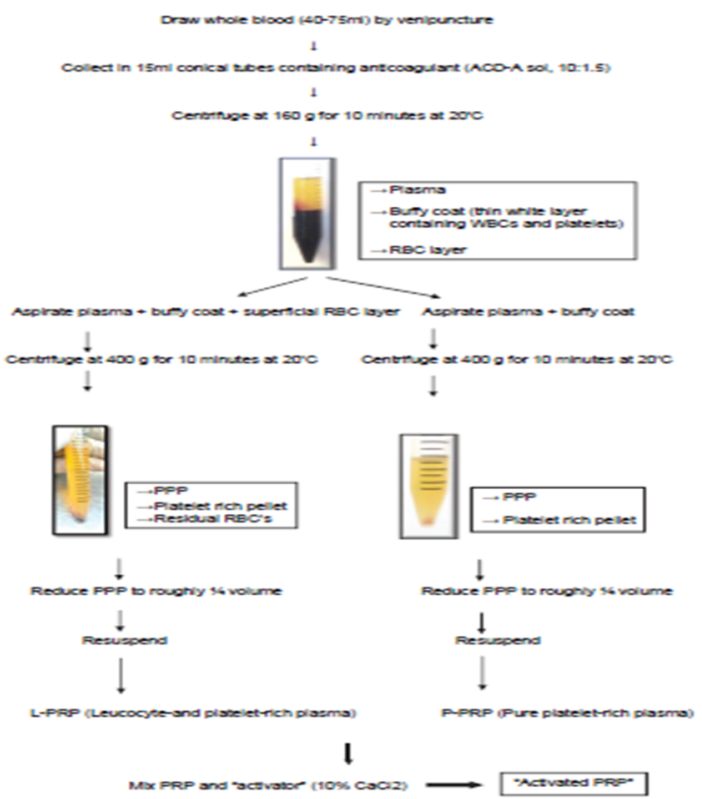

Flowchart Depicting The Conventional Method Of Prp Preparation Used In The Study

Types Of Platelet Preparations

P-PRP(Pure platelet-rich plasma)

The P-PRP concentrate consists of an undetermined fraction of buffy coat, containing a large number of platelets, but most leucocytes are not collected.

L-PRP(Leucocyte- and platelet-rich plasma)

L-PRP consists of most of the platelets, along with leucocytes and some residual RBCs, suspended in fibrin-rich plasma.

The manual PRP preparation process (as described above) is not clearly defined, it might randomly lead to P-PRP or L-PRP.

P-PRF (Pure platelet-rich fi brin)

The term PRF is used synonymously with platelet-rich fibrin matrix (PRFM). When P-PRP is mixed with activator and allowed to incubate for some time, a stable PRFM clot can be collected.

L-PRF (Leucocyte- and platelet-rich fi brin)

Here, blood is collected without any anticoagulant and immediately centrifuged. A natural coagulation process then occurs and three layers are formed: the RBC base layer, acellular plasma top layer and L-PRF clot in the middle, which harvests platelet and leucocyte growth factors into the fibrin matrix.

There is no biochemical modification of the blood, i.e. no anticoagulants, thrombin or $\mathrm{CaCl} 2$ are required.

\section{MATERIALAND METHOD}

In this open label study conducted at a tertiary care centre of north India , 25 patients with chronic non healing ulcers preferably on the lower leg and feet were enrolled. Patients presenting with ulcers elsewhere on the body were not included in the study.

Each patient was treated with PRP prepared (by the conventional method) with their own blood which was injected with a 26 gauge needle in the periphery and floor of the ulcer at a weekly interval for 1 month. Subjective Response was assessed by comparing the VAS scoring done by patients at the start and end of treatment . Objective Response was assessed by comparing photographs at each visit and by measuring the size of the ulcer in all it's dimensions.

Regular daily dressing with paraffin gauge and an antibiotic cream containing mupirocin was done for all patients. Patients having co morbidities such as diabetes mellitus, hypertension, hyperlipidemia were also given treatment for the same.

\section{RESULTS}

Out of 25 patients, 15 were males and 10 were females. The age group varied from 25-65 years. The site of ulcer most commonly found in patients was the ball of great toe (10 patients), leg ( 9 patients) and other parts of foot and leg ( 6 patients). 12 out of 25 patients had diabetes mellitus, of which 4 had associated hypertension and hyperlipidemia. The other 13 were free of co morbidities.

The most common etiology for ulcers was diabetes (12 patients), vascular insufficiency (6 patients), leprosy (5 patients) and traumatic (2 patients).

Signicant improvement in the visual analogue score (VAS) scored by the patients themselves was seen at the end of the therapy. The score improved from 3 in $60 \%$ patients at the start of therapy to 9 in $70 \%$ and 10 in $30 \%$ patients at the end of therapy. There was excellent improvement noted in the ulcers in terms of reduction in size, appearance of granulation tissue and reduced induration by $75 \%$ in 19 patients while in remaining 6 patients there was only $50 \%$ improvement seen.

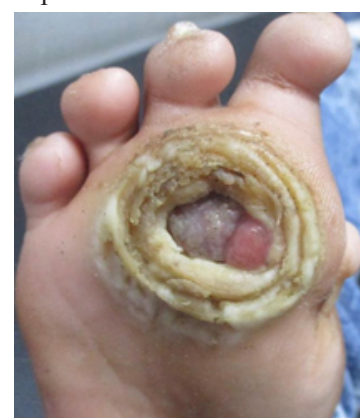

Figure 1a: Chronic Ulcer (foot)

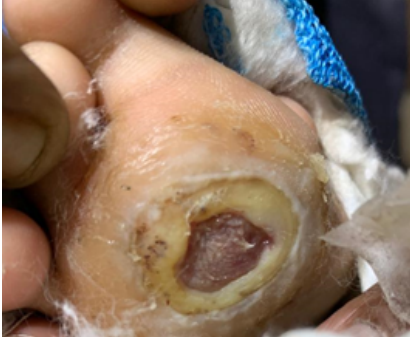

Figure1b: Same Ulcer At The End Of Therapy (4 Weeks)
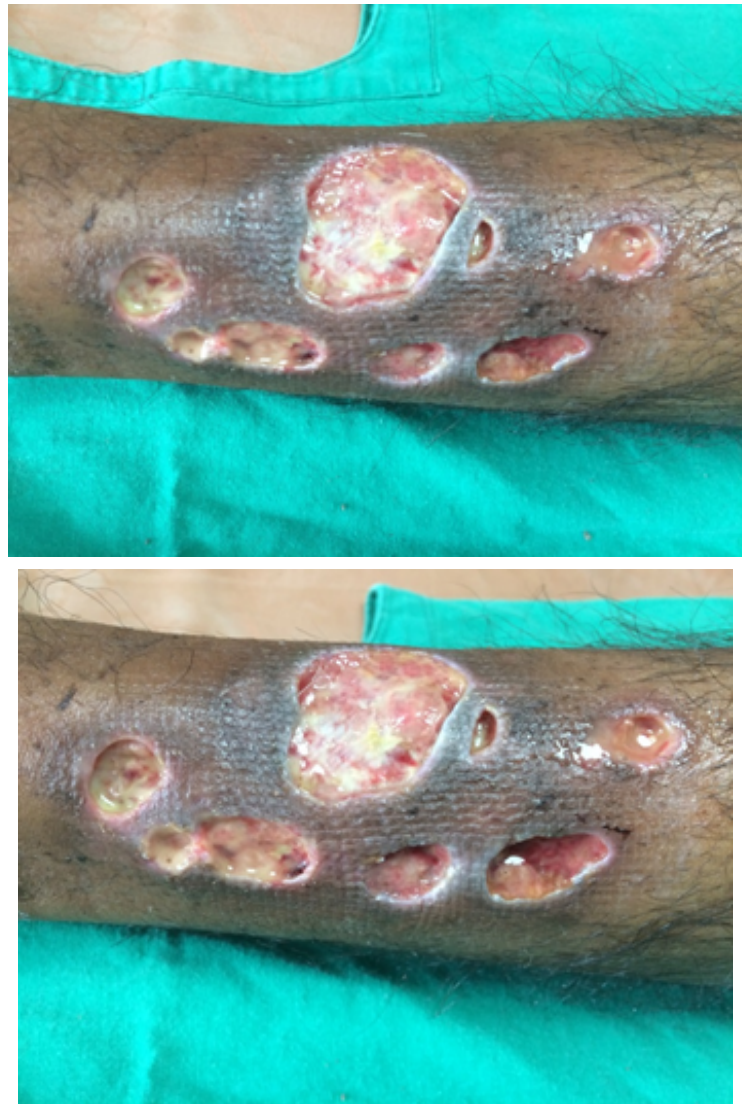

Figure 2 A\&b: Non Healing

Chronic Ulcers On Lower Leg 


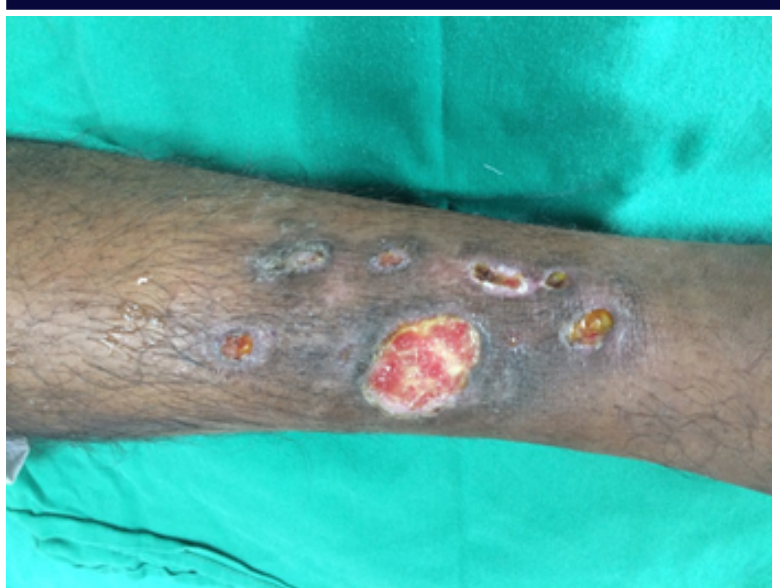

Figure 2 C: Ulcer At 2 Weeks

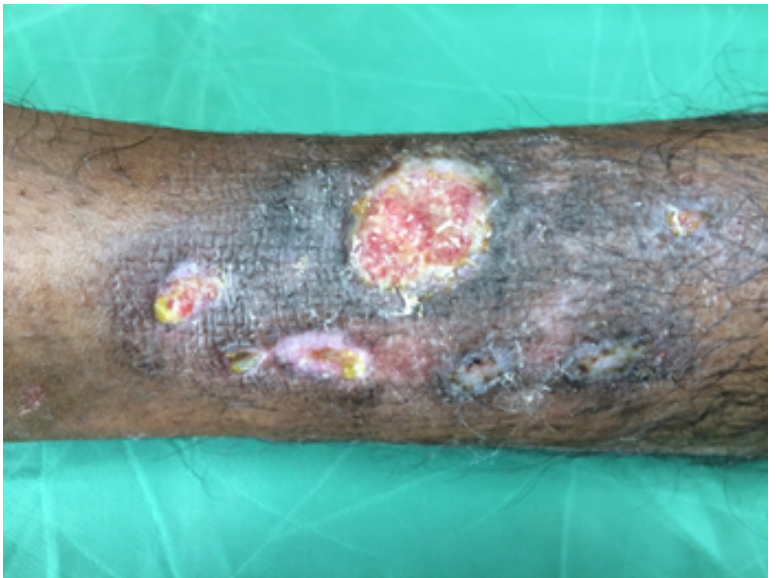

Figure 2d: Ulcer At 3 Weeks
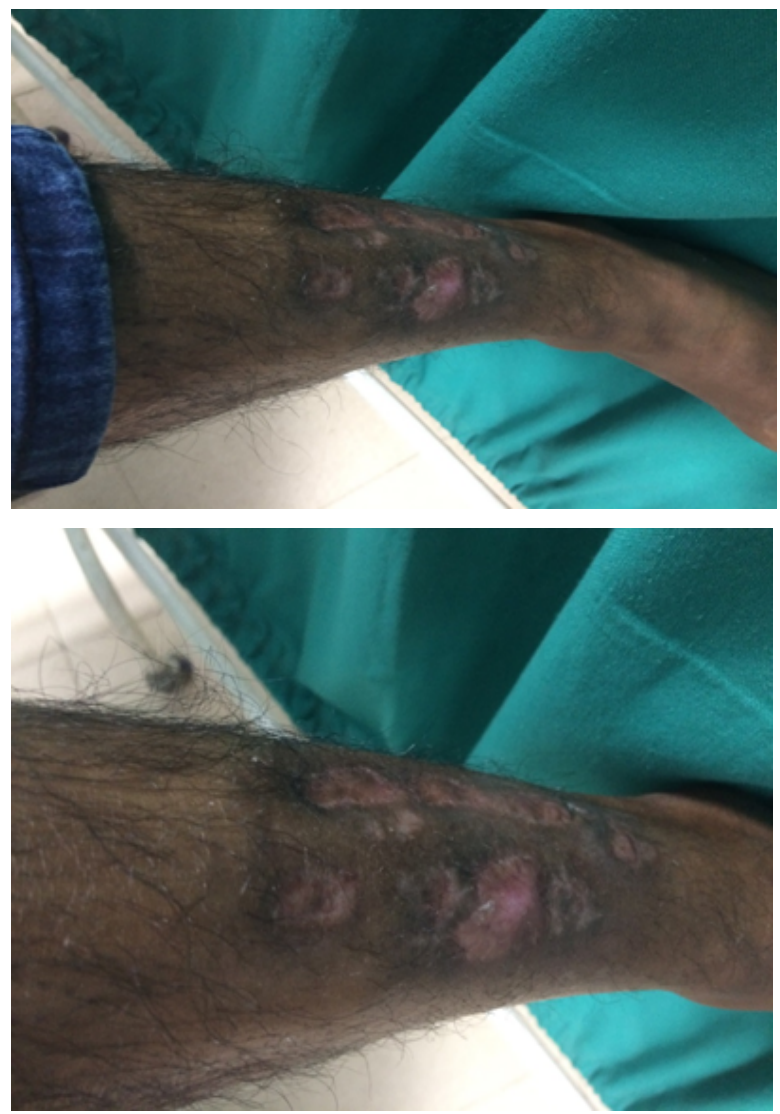

Figure 2e\&f : Ulcer At 4 Weeks

DISCUSSION

Due to the novelty of PRP and its more recent discovery, few studies have been conducted on its efficacy in human subjects.

PRP has been found to be effective in several case control studies in addition to several non-controlled clinical trials. Only one prospective, randomized, controlled clinical trial has been reported regarding the use of autologous PRP for the treatment of diabetic foot ulcers. There are studies supporting the role of PRP.

In a study by McAleer et al. (2006) found that the use of autologous PRPwas successful in healing a chronic lower extremity wound in a case study of a 57-year-old man with type 2 diabetes and a wound of six months duration. Complete closure of the ulcer was achieved by the fourth week of treatment with PRP. The finding is similar to that found in our study.

Salemi et al. (2008) ${ }^{10}$ was a more recent case study evaluating the effectiveness of a combination of autologous adipose tissue and PRP in a lower extremity ulcer of three years duration in a non-diabetic 65year-old male patient. This study lasted for four weeks with follow-up at one, three, six, and 15 months. At a 15-month followup, the wound had healed completely with regained functioning of the limb and a greatly enhanced quality of life reported by the patient.

Margolis et al. (2001) ${ }^{11}$ was a retrospective cohort study devised to estimate the effectiveness of platelet releasate (PR) in the treatment of diabetic neuropathic foot ulcers.

Crovetti et al. (2004) $)^{12}$ published a prospective non-blinded study regarding the efficacy of platelet gel (PG) in healing cutaneous chronic wounds.

The protocol for this study consisted of once-weekly PG applications of either autologous or homologous origin. At the time of the study publication, nine patients had healed completely, two went on to receive cutaneous grafts, four had stopped treatment, and nine had responded partially.

Driver et al. (2006) ${ }^{13}$ carried out the first reported prospective, randomized, controlled multicenter trial in the United States regarding the use of autologous PRP for the treatment of diabetic foot ulcers.

While are a few which contradict the use of PRP, Margolis et al $(2002)^{14}$ carried out a retrospective cohort study of 31,106 individuals suggesting that these therapies had little effect on the study results. The authors of this study attributed this effect to selection bias, with physicians likely selecting those patients doing poorly to receive adjuvant care.

The reason for choosing PRP was for the process being autologous has no adverse effects, is reasonable and PRP represents a greater similarity to the natural healing process, with the application of multiple growth factors in their biologically determined ratios.

\section{CONCLUSION}

Despite the many advances in the treatment of chronic ulceration, this common complication continues to devastate the patients.

It is important to motivate both patients and clinicians to attempt these more advanced treatment modalities, as treatment with growth factors may result in faster healing. RPR should be sought as a treatment modality for such ulcers for it many benefits enumerated in the article.

\section{REFERENCES}

1. Marx RE, Garg AK. Dental and Craniofacial Applications of Platelet-Rich Plasma. Chicago: Quintessence Publishing; 2005.

2. Phillips T. Ulceras. In: Bolognia JL, Jorizzo JL, Rapini RP, editors. Dermatologia. 2nd ed. London: Mosby; 2008:1597-1614

3. Nurden AT, Nurden P, Sanchez M, Andia I, Anitua E. Platelets and wound healing. Front Biosci. 2008:13:3532-3548

4. Sanchez M, Andia I, Anitua E, Sanchez P. Platelet rich plasma (PRP) biotechnology: concepts and therapeutic applications in orthopedics and sports medicine. In: Agbo EC editor. Innovations in Biotechnology. Rijeka, Croatia: InTech; 2012:113-138.

5. Steed DL. The role of growth factors in wound healing. Surg Clin North Am 1997; 77:575-86.

6. Marx RE, Carlson ER, Eichstaedt RM, Schimmele SR, Strauss JE, Georgeff KR Platelet-rich plasma: Growth factor enhancement for bone grafts. Oral Surg Oral Med Oral Pathol Oral RadiolEndod 1998;85:638-46.

7. Marx RE. Platelet-rich plasma: Evidence to support its use. J Oral Maxillofac Surg 2004;62:489-96.

8. American Association of Blood Banks technical manual committee. Method 6.11: Preparation of platelets from whole blood. In: Vengelen-Tyler V, editor. AABB 
Technical Manual, 13th ed. Bethesda (MD): American Association of Blood Banks; 1999.p. 725 .

9. McAleer JP, Sharma S, Kaplan EM, Persich G. Use of autologous platelet concentrate in a nonhealing lower extremity wound. Adv SkinWound Care. 2006;19(7):354-63.

10. Salemi S, Rinaldi C, Manna F, Guarneri GF, Parodi PC. Reconstruction of lower leg skin ulcer with autologous adipose tissue and platelet-rich plasma. J Plast ReconstrAesthet Surg. 2008;61(12):1565-7

11. Margolis DJ, Kantor J, Santanna J, Strom BL, Berlin JA. Effectiveness of platelet releasate for the treatment of diabetic neuropathic foot ulcers. Diabetes Care. 2001;24(3):483-8.

12. Crovetti G,Martinelli G, IssiM, et al. Platelet gel for healing cutaneous chronic wounds. Transfus Apher Sci. 2004;30(2):145-51.

13. Driver VR, Hanft J, Fylling CP, Beriou JM, Autologel Diabetic Foot Ulcer Study Group. A prospective, randomized, controlled trial of autologous platelet-rich plasma gel for the treatment of diabetic foot ulcers. Ostomy Wound Manage. 2006;52(6):68-70, 72, 74 treatment
passim.

14. Margolis DJ, Allen-Taylor L, Hoffstad O, Berlin JA. Diabetic neuropathic foot ulcers: the association of wound size, wound duration, and wound grade on healing. Diabetes Care. 2002;25(10):1835-9. 